\title{
Stanze dipinte. L'Inamoramento de Orlando come allegoria dell'Amicizia nella Sala del bacio nel Palazzo del Giardino a Parma
}

\section{Angelo Maria Monaco}

To cite this article: Angelo Maria Monaco (2019): Stanze dipinte. L'Inamoramento de Orlando come allegoria dell'Amicizia nella Sala del bacio nel Palazzo del Giardino a Parma, Italian Studies

To link to this article: https://doi.org/10.1080/00751634.2019.1587232

曲 Published online: 19 Mar 2019.

Submit your article to this journal

View Crossmark data $\nearrow$ 


\title{
Stanze dipinte. L'Inamoramento de Orlando come allegoria dell'Amicizia nella Sala del bacio nel Palazzo del Giardino a Parma
}

\author{
Angelo Maria Monaco \\ Lecce - Accademia di Belle Arti
}

\begin{abstract}
The essay analyses the iconography of the sixteenth-century frescoes by the Mannerist painter Bertoja in the 'Sala del bacio' of the 'Palazzo del Giardino', Parma. The series is interpreted as an allegory on the humanist theme of 'Friendship', seen as a Boiardan, epic, and aristocratic topic by members of the so-called 'Republic of Letters', of which Ottavio Farnese, patron of the frescoes, was a prominent member. The frescoes provide evidence of the fortunes of Boiardo's poem beyond the limits of the period's illustrated editions, displaying a different dimension of the poem's visual reception.
\end{abstract}

\section{KEYWORDS}

Palazzo del Giardino (Parma); Sala del bacio (Parma); Patrons and Painters; Iconography; Jacopo Zanguidi (Bertoja); 'Repubblica delle Lettere'; Friendship

I poemi di Boiardo e di Ariosto, come più tardi sarebbe accaduto a quello di Tasso, godettero nell'ambito delle arti figurative, di una fortuna assai precoce. Le articolate vicende narrate nelle stanze di versi, non solo offrirono a pittori e scultori una copiosa varietà di soggetti da riprodurre su opere mobili di piccolo e di medio formato, ma risultarono assai adeguate per decorare vaste superfici ad affresco in nobili dimore, appartenenti a colti patroni delle arti, esponenti di quella 'République des Lettres' su cui si è soffermato a lungo Marc Fumaroli. ${ }^{1}$ Come argomenta Giovanna Rizzarelli, in questa stessa sede editoriale, il processo di fortuna dei medesimi componimenti fu accelerato dalla circolazione delle edizioni a stampa illustrate, in un rispecchiamento inevitabile tra poesia e parola fedele al canone oraziano dell'ut pictura poësis.

Nell'ambito delle arti figurative, se da un lato molte opere visualizzano singoli personaggi o scene desunte dalle stesse fonti, con una funzione meramente iconografica e narrativa, dall'altro, quanto più è sofisticata la cultura della committenza a cui esse sono destinate, tanto maggiore è la componente allegorica che occultano. Di questo tipo sono senza dubbio gli affreschi nella 'Sala del bacio' del 'Palazzo del Giardino' di Parma, in cui la scelta programmatica di ricorrere a una porzione ben precisa del poema di Boiardo, il canto settimo del terzo libro, il cui tema proemiale è espressamente dichiarato e individuato nel topos umanistico dell'Amicizia, sottende l'esigenza di elevare su un piano etico e morale le rocambolesche avventure affrontate da Orlando. Laddove il paladino, sedotto e distratto per incantamento dall'alto officio della cavalleria cui è destinato, può far ritorno a onorarlo grazie all'intervento di alleati fedeli.

Secondo tale prospettiva, allora, l'intero ciclo parmense, il cui rapporto diretto con la narrazione in versi si tenta di seguito di analizzare a fondo, esalterebbe il topos ciceroniano dell'amicizia, inteso come virtus peculiare di colui che, con le armi, con l'ingegno, o intessendo le più opportune alleanze, è tenuto a compiere il proprio destino di principe 'illuminato' e di 
condottiero. Una metafora chiara che colloca il committente degli affreschi, Ottavio Farnese, in una posizione specifica rispetto al complesso scenario politico e culturale in cui si ritrovò a governare. $^{2}$

Tra il 1562 e il 1570, Girolamo Mirola e Jacopo Zanguidi, detto il Bertoja, affrescarono due stanze al piano nobile del Palazzo ducale di Parma. Nell'ambito della campagna di rinnovamento del cosiddetto 'Palazzo del Giardino' voluta da Ottavio Farnese, ${ }^{3}$ i due frescanti emiliani furono chiamati a trarre i soggetti non già dalle 'favole degli antichi', come era assai diffuso nella fervida stagione della Maniera e a Caprarola, residenza del fratello minore di Ottavio, 'il gran cardinale' Alessandro, ma dai poemi epici divenuti più alla moda in quel tempo. Concepiti come ambienti ibridi, destinati tanto alla vita privata del committente quanto al ricevimento di ospiti illustri, le due stanze sono note come 'Sala di Alcina', con scene tratte dal Furioso, e come 'Sala del bacio', con scene tratte dall'Inamoramento de Orlando di Matteo Maria Boiardo. Intorno al 1628 tale ciclo epico sarebbe stato portato a compimento dal pittore Alessandro Tiarini con la 'Sala di Erminia' d'ispirazione tassiana, completando tipologicamente un sistema iconografico di certo carico di implicazioni allegoriche volte a celebrare gli interessi culturali, i fasti e la missione politica dei Farnese. La scelta di ricorrere ai tre poemi in un arco cronologico di un cinquantennio, non solo ratifica il godimento di un equivalente livello di stima per i rispettivi autori, ma soprattutto dimostra le potenzialità di riconversione delle ottave epiche in linguaggio figurativo.

Già apprezzati all'epoca del Grand Tour, gli affreschi della 'Sala del bacio' godono di una letteratura critica eterogenea, accomunata da una raffinata qualità esegetica ma anche dalla mancata esplorazione, molto spesso, di due aspetti invece rilevanti: da un lato, la lettura ecfrastica della porzione terminale degli affreschi e, dall'altro, la loro connessione con l'argomento specifico del canto su cui si fondano. ${ }^{4}$

L'Inamoramento de Orlando fu letteralmente schiacciato dal successo del poema di cui costituisce l'antefatto, cioè il Furioso. Ormai indagato nei minimi risvolti e da varie prospettive metodologiche, il poema ariostesco rende straordinari quei casi in cui esso non costituisca il nucleo tematico ed esclusivo di un contesto iconografico di soggetto epico nel Cinquecento. Così accade nel 'Palazzo del Giardino',

\footnotetext{
${ }^{2}$ Desidero ringraziare Giovanna Rizzarelli, per avermi proposto di partecipare al progetto editoriale già durante il periodo di collaborazione trascorso presso il CTL (Centro di Elaborazione Informatica di Testi e Immagini nella Tradizione Letteraria) della Scuola Normale Superiore di Pisa, tra il 2014 e il 2015 e per aver accolto il saggio in questa sede; Massimiliano Rossi e Alice Rossi per averlo discusso in anteprima. Un sentito ringraziamento va inoltre al Comando Provinciale dei Carabinieri di Parma, che ha sede nel 'Palazzo del Giardino', in particolare al Colonnello Salvatore Altavilla e al Tenente Colonnello Andrea Pacchiarotti, e all'Ufficio Patrimonio Artistico - Civiche Collezioni Patrimonio del Comune di Parma per avermi concesso di realizzare le riprese fotografiche degli affreschi che qui si pubblicano.

${ }^{3}$ Su cui si veda La reggia di là dall'acqua: Il palazzo e il giardino dei duchi di Parma, a cura di Giovanni Godi (Milano: Franco Maria Ricci, 1991).

${ }^{4}$ Riferimenti agli affreschi nell'ambito della letteratura periegetica si riscontrano in Nicodemus Tessin il giovane, per la seconda volta in voyage d'étude in Italia tra il 1687 e il 1688, su cui si veda Diane De Grazia, Bert W. Meijer, 'Mirola and Bertoia in the Palazzo del Giardino, Parma', The Art Bulletin, 69.3 (1987), 395-406, che utilizzò come guida il Viaggio pittoresco di Giacomo Barri (Venezia: Giacomo Herz, 1670), la cui edizione critica moderna è di Angelo Maria Monaco, Giacomo Barri e il suo 'Viaggio pittoresco d'Italia': Gli anni a Venezia di un peintre-graveur scrittore d'arte (Firenze: Edifir, 2014), pp. 95 e 121 n. 98. In epoca moderna si sono occupati del ciclo di affreschi, tra gli altri e secondo molteplici prospettive: Augusta Ghidiglia Quintavalle, II Bertoja (Parma: Cassa di Risparmio di Parma, 1963); Silvia De Vito Battaglia, 'Leggende cavalleresche nel Palazzo del Giardino a Parma', Aurea Parma, 56.1 (1972), 3-16; Vittorio Sgarbi et al., 'Voluptas', FMR (Franco Maria Ricci), 22 (1984), 65-86, dove però è presentata come inedita l'individuazione della fonte iconografica, a p. 70; Roberto Venturelli, La corte farnesiana di Parma (1560-1570): Programmazione artistica e identità culturale (Roma: Bulzoni, 1999), in part. pp. 67-86 per il ruolo svolto da Bernardo Tasso, autore dell'Amadigi nel 1560, come inventor del programma iconografico; Andrea Ghirarduzzi, 'Il Furioso per immagini: uno studio iconografico e iconologico degli affreschi del Palazzo Ducale del Giardino a Parma', News-Art. Notizie dal mondo dell'arte, 1 marzo 2017, <http://news-art.it/news/la-maniera-emiliana-nei-dipinti-del-palazzo-ducale-delgi.htm> [accesso del 10 dicembre 2018]. Di Bert W. Meijer, si veda inoltre Parma e Bruxelles: Committenza e collezionismo farnesiani alle due corti (Milano: Amilcare Pizzi, 1988), dove è restituito il merito dell'individuazione della fonte alla De Vito Battaglia, 'Leggende cavalleresche', p. 29 n. 25. Sulla decorazione del palazzo sono in corso le ricerche di Alice Rossi che ha discusso nel 2018, presso la Facoltà di Beni Culturali dell'Università del Salento, la tesi di Dottorato: 'Immagini e formule dell'encomio. Variazioni nella tipologia del sovrano carismatico fra Cinque e Seicento'. Per la fortuna iconografica degli altri due poemi è assai vasta la bibliografia a disposizione, qui sia sufficiente fare riferimento, per il Furioso a L'Orlando Furioso nello specchio delle immagini, a cura di Lina Bolzoni (Roma: Istituto della Enciclopedia Italiana, 2015), per la Liberata a L'arme e gli amori: La poesie di Ariosto, Tasso e Guarini nell'arte fiorentina del Seicento, catalogo della mostra a cura di Elena Fumagalli, Massimiliano Rossi, e Riccardo Spinelli (Livorno: Sillabe, 2001).
} 
AF: Età dell'oro (allegoria)

AF I: AETAS FOELICIOR (iscrizione)

III

A: raduno dei cavalieri

B: bosco incantato

C: incantesimi

D: Ruggero e Gradasso trasportati nel fiume Riso

TSQV: TRAHIT SUA QUEMQUE VOLUPTAS (iscrizione)

E: festa nella reggia di cristallo/ rottura dell'incantesimo

F: liberazione dei cavalieri/riemersione dal lago

G: Orlando sfidato da Gradasso/nano a cavallo

$\mathrm{H}$ : irruzione della guerra

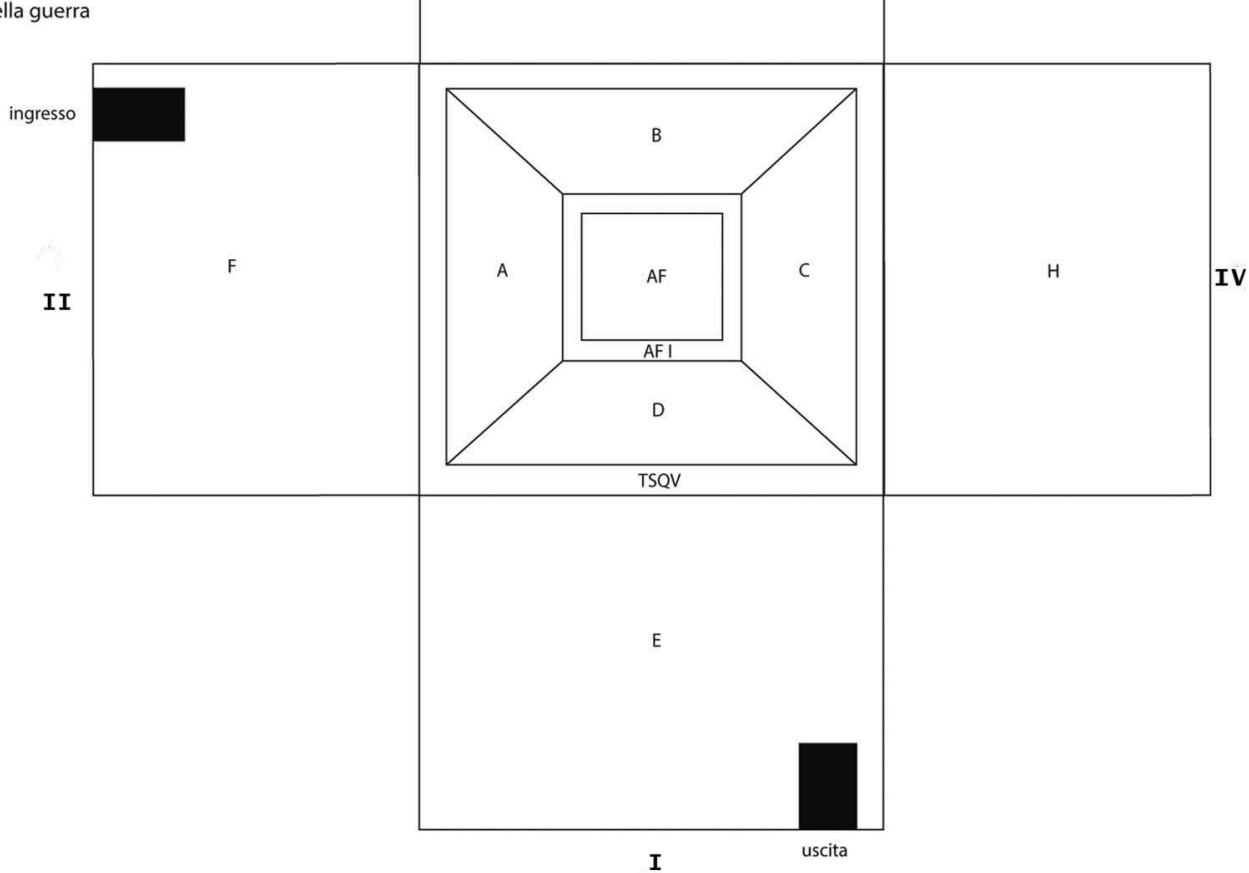

Figura 1. Schema iconografico della 'Sala del bacio'. Elaborazione grafica A.M.M.

Crediti fotografici: Comune di Parma, Civiche Collezioni d'Arte - Ufficio Patrimonio Artistico

dove appunto la scelta di ricorrere al Furioso per la decorazione degli ambienti è affatto prevaricante rispetto a quella di accostarlo ai rispettivi testimoni 'ascendente' e 'discendente', cioè l'Inamoramento e la Liberata.

Secondo un canone iconografico di ripartizione ordinata della superficie destinata ad accogliere i partimenti decorativi, la 'Sala del bacio' è strutturata in tre registri iconografici sovrapposti, intervallati da due iscrizioni, impostati su un'alta zoccolatura perimetrale. Nella volta a padiglione svetta una campitura centrale incorniciata con stucchi che incorpora il titulus di matrice ovidiana 'Aetas foelicior'; sulle quattro vele che compongono la volta è sviluppato un registro iconografico superiore a fregio continuo, a cui fa da base un cornicione d'imposta che ingloba una sentenza virgiliana e che rimarca la ripartizione con il registro di immagini inferiore sviluppato sulle pareti verticali (Figura 1: numerate da I a IV). Come in un finto loggiato che si apre verso l'esterno, corre alla base della stanza una zoccolatura dipinta con un fregio di elementi architettonici aggettanti in prospettiva, su specchiature di finti marmi alternati a mascheroni. Mentre il riquadro centrale è dipinto con il mito arcadico dell'Aetas foelicior (Figura 2), ${ }^{5}$ il secondo e il terzo registro di affreschi sono scanditi in quattro sezioni, ognuna

${ }^{5}$ Sulle fonti iconografiche utilizzate dai pittori per la raffigurazione del soggetto (ad esempio la xilografia di Bernard Salomon nell'edizione lionese del 1557 delle Metamorfosi), si rimanda ancora una volta a Meijer, Parma e Bruxelles, p. 29 n. 25. 


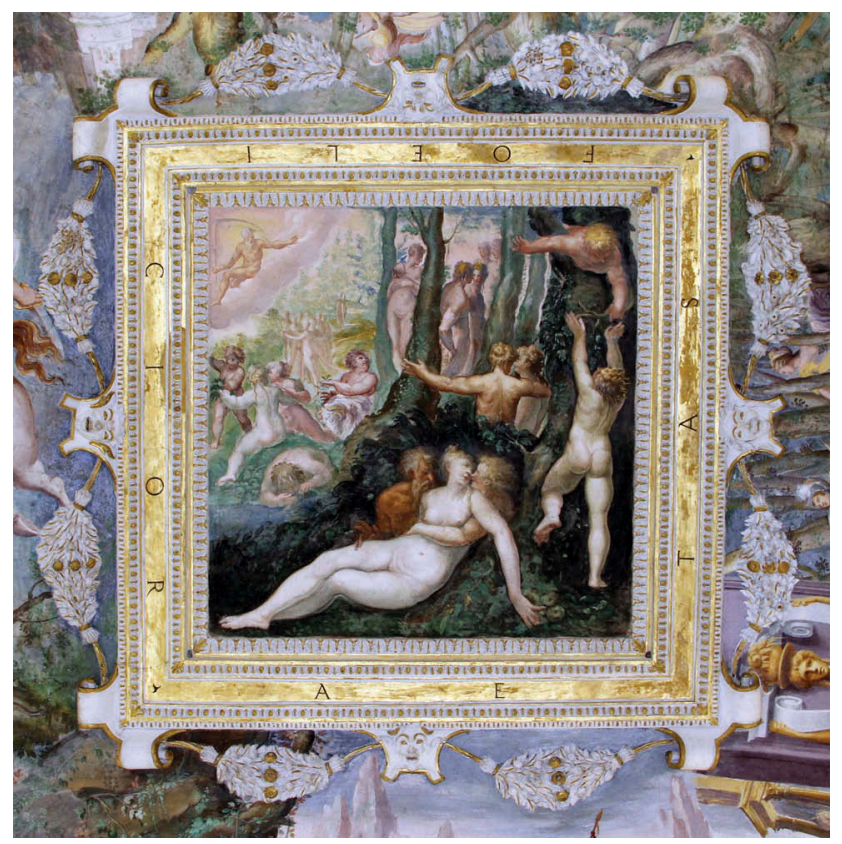

Figura 2. 'Sala del bacio', volta, riquadro centrale: Aetas foelicior, allegoria dell'Età dell'oro. Su concessione del Comune di Parma, Civiche Collezioni d'Arte - Ufficio Patrimonio Artistico.

Crediti fotografici: Comune di Parma, Civiche Collezioni d'Arte - Ufficio Patrimonio Artistico

recante un episodio del canto settimo del terzo libro dell'Inamoramento de Orlando. ${ }^{6}$ In ogni sezione, gli episodi raffigurati vedono protagonisti alcuni paladini alle prese con una missione ben precisa: la liberazione di Orlando, rimasto vittima di un incantesimo che lo distoglie dall'alto e nobile officio della cavalleria.

La composizione è di grande eleganza e di alta qualità stilistica. Siamo nel languido clima della Maniera parmense, figlia della lezione magistrale di Correggio e di Parmigianino che proprio in città avevano lasciato il frutto della loro pittura più sofisticata (dalla 'Camera della Badessa' Giovanna da Piacenza, nel Convento di San Paolo, agli affreschi per San Giovanni Evangelista) ${ }^{7}$; in un contesto del tutto laico che in apparenza ignora le istanze della Controriforma, cedendo ancora alle lusinghe del mito, dell'epos e dell'eros impiegati in chiave allegorica, affatto vulcanizzati della loro carica profana. ${ }^{8}$ La complessa iconografia amplifica lo spazio finito della stanza illudendo con grande efficacia l'osservatore e consentendogli di leggere il 'testo' dipinto come se si trattasse delle pagine di un libro, con la medesima agilità favorita dalla metrica dei versi, qui tradotti in una narrazione che si srotola altrettanto ritmicamente. Come sostenne Jacob Burckhardt a proposito delle caratteristiche stilistiche dei poemi di Pulci e Boiardo, pur senza fare riferimento agli affreschi di Parma dei quali sembra quasi offrire una lettura ecfrastica assai calzante, 'their composition is not that of a great historical picture, but rather that of a frieze, or of some rich festoon entwined among groups of picturesque figures'. ${ }^{9}$ Consapevoli dell'azione che compiono, i pittori trasformano le stanze di versi in

\footnotetext{
${ }^{6}$ Per una lettura puntuale dell'episodio e delle sue fonti classiche si rimanda a Cristina Zampese, 'Or si fa rossa or pallida la luna': La cultura classica nell'Orlando Innamorato (Lucca: Pacini Fazzi, 1994), pp. 119-25.

${ }^{7} \mathrm{Si}$ fa riferimento a opere arcinote dei due pittori emiliani per le quali, tuttavia, si rimanda, ad esempio, a Elisabetta Fadda, Come in un rebus. Correggio e la camera di San Paolo (Firenze: Olschki, 2018); per gli affreschi di Parmigianino nella chiesa di San Giovanni Evangelista, si veda Mario di Giampaolo e Elisabetta Fadda, Parmigianino (Sant'Angelo di Romagna: Keybooks, 2002).

${ }^{8}$ Un livello di significato alchemico/ermetico negli affreschi alla luce del tema del bacio e della coniunctio è ipotizzato da Maurizio Fagiolo Dell'Arco in II Parmigianino: Un saggio sull'ermetismo nel Cinquecento (Roma: Bulzoni, 1970), p. 133 n. 38.

${ }^{9}$ Jacob Burckhardt, Die Kultur der Renaissance in Italien (Schweighauser: Basel, 1860); qui citato dall'edizione in inglese The Civilization of the Renaissance in Italy, trad. di S. G. C. Middlemore (London-New York: Phaidon Press, 1944), p. 196.
} 
stanze dipinte, costellando la superficie pittorica di chiavi iconografiche utili tanto a scandire la temporalità e la progressione degli eventi della narrazione, quanto a riattivare nella memoria dell'osservatore le ottave del poema, svincolando le scene da una struttura geometrica di fondo, ma proprio intrecciandole come in un 'fregio' continuo o in 'un ricco festone'.

Posto il sigillo iconografico e allegorico nella volta, dove, come ha argomentato Roberto Venturelli alla luce di un passo della Theologia di Marsilio Ficino, ${ }^{10}$ Venere è percepibile come incarnazione della Voluptas, la narrazione tratta da Boiardo trova il suo incipit nella vela collocata sulla parete qui definita seconda. In questa scena i paladini sono costretti a separarsi in due gruppi costituiti dal solo Mandricardo, qui ritratto di tergo a cavallo in atto di allontanarsi, e da Ruggero, Gradasso, Brandimarte e Fiordelisa, i quali, insieme, dopo aver letto un testo ammonitore e ingannevole iscritto su un cartiglio a doppia facciata dispiegato da una figura femminile collocata in un'edicola, ${ }^{11}$ si inoltrano in una fitta selva alla ricerca di Orlando rapito dalle Naiadi (Inamoramento, III. 7. 12-14). Il punto di partenza della composizione è rintracciato allora in una fase avanzata del poema, quando è già resa manifesta la rilevanza del concetto di Amicizia intesa come collante della solidale società cavalleresca, fedele a un'etica e a una deontologia che accomuna i paladini degni di tale officio, esplicitato con versi icastici nelle stanze proemiali del canto:

Più che il tesoro e più che forza vale,

Più che il dilecto assai, più che l'onore,

El bono amico e compagnia leale:

$\mathrm{E}$ a doi che insieme se portano amore,

Magior li pare el ben, minor el male,

Potendo apalesar l'un l'altro el cuore;

E ogni dubio che acade, o raro o spéso,

Poterlo ad altrui dir come a sé stesso.

(Inamoramento, III. 7. 1)

Il racconto visivo procede lungo le vele collocate sulle pareti terza e quarta, dove figure prodigiose fuoriuscite dai tronchi di alberi recisi dai paladini per farsi strada, rapiscono i loro liberatori portandoli in aria per farli poi sprofondare negli abissi del fiume del Riso, dov'è già Orlando. Una ninfa dai capelli d'oro, priva di veli, seduce Ruggero convinto di salvarla da una metamorfosi arborea incombente, di memoria ovidiana (Inamoramento, III. 7. 17. 1-6 e 18. 1-4). Un maestoso destriero su cui è montato Gradasso (Inamoramento, III. 7. 22 e 27) si libra in volo facendo precipitare il cavaliere come il Fetonte di Michelangelo nell'incisione di Beatricetto, di certo nota al pittore. ${ }^{12}$ Fedele all'iconografia fluviale, il fiume del Riso è personificato all'antica, in due punti ben precisi della composizione, per significare i suoi stadî di sorgente e di foce, oltre che per dare le coordinate di apertura e chiusura di una sezione compiuta del racconto. La prima volta il fiume del Riso è ritratto come un giovane, riverso su un'anfora da cui fuoriesce un potente getto d'acqua, nel punto d'accesso alle sue stesse profondità, dove i paladini rapiti affondano (Figura 3); la seconda volta è invece replicato come un vecchio con capelli e barba lunghissima ma ancora aitante, quindi come foce, là dove si conclude il capitolo relativo alle vicende acquatiche, sulla parete di rimpetto (Figura 5). La

\footnotetext{
${ }^{10}$ Qui sia sufficiente richiamare il rapporto rintracciato dallo studioso tra abbandono alla Voluptas terrena e la perdita del sé, individuato da Venturelli come idea di fondo dell'iconografia della sala: vedi Venturelli, La corte farnesiana, p. 34.

${ }^{11}$ I cui versi, non riprodotti nell'affresco, si leggono in Inamoramento, III. 7. 13: '“Disio di chiara fama, isdegno e amore | Trovano aperta a sua voglia la via". | Questi dui versi avea scritti di fuore, | Poi dentro in cotal modo se legìa: | "Amore, isdegno e il desiar onore, | Quando hano preso l'animo in balia, | Lo sospingono avanti a tal fracasso, | Che poi non trova a ritornar el passo"'. Tutte le citazioni sono tratte da Matteo Maria Boiardo, L'Orlando innamorato: L'inamoramento de Orlando, a cura di Andrea Canova, 2 voll. (Milano: Rizzoli, 2011).

${ }^{12} \mathrm{Si}$ fa riferimento alla nota incisione a bulino di Nicolas Beatrizet (detto il 'Beatricetto') di cui un esemplare a Vienna, Albertina, 1540 ca., realizzata da un disegno a carboncino su carta di Michelangelo, 1532 ca., (Windsor Castle, R.L.) per Tommaso de' Cavalieri. Si veda Evelina Borea, Lo specchio dell'arte italiana: Stampe in cinque secoli, 4 voll., (Pisa: Edizioni della Normale, 2009), I, 123 e II, capitolo secondo, Figure 1 (p.n.n.).
} 


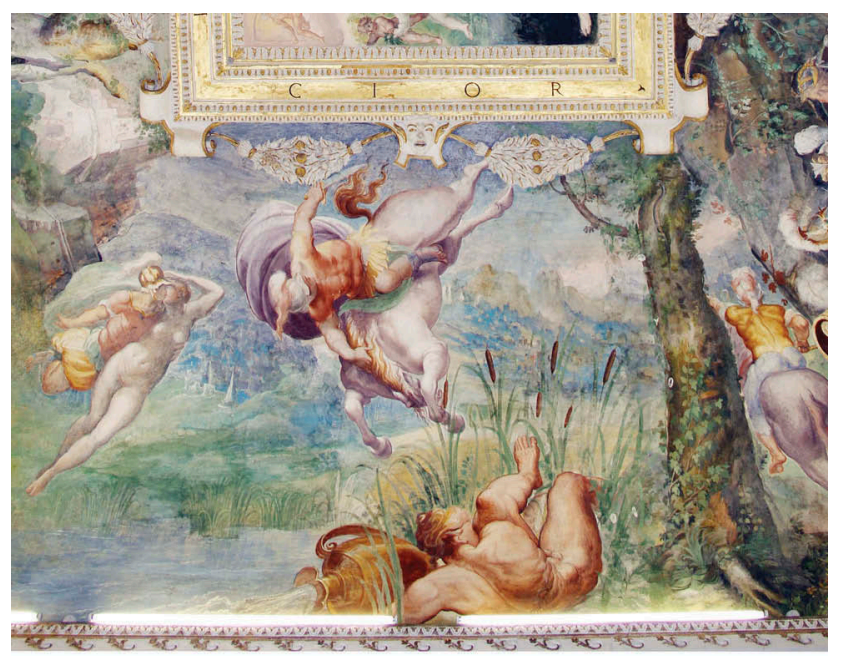

Figura 3. 'Sala del bacio', vela sulla parete I: Ruggero trasportato da una Naiade/Gradasso precipita nel lago/Personificazione del fiume del Riso come sorgente. Su concessione del Comune di Parma, Civiche Collezioni d'Arte - Ufficio Patrimonio Artistico. Crediti fotografici: Comune di Parma, Civiche Collezioni d'Arte - Ufficio Patrimonio Artistico

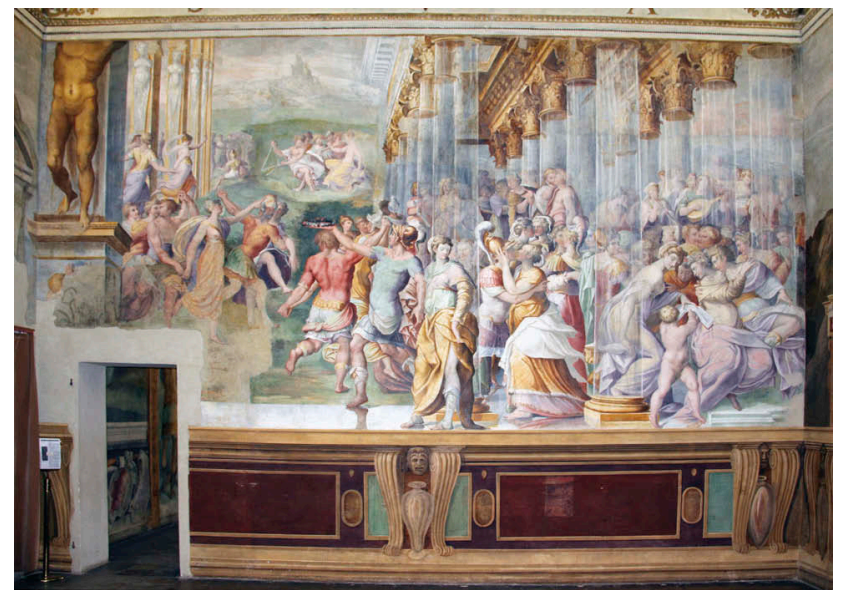

Figura 4. 'Sala del bacio', parete I: la festa nel palazzo dalle colonne di cristallo; Fiordelisa (a sinistra con elmo e con un seno scoperto) libera Ruggero; (al centro) Brandimarte reca in mano una coroncina come antidoto contro l'incantesimo; Orlando (a destra con elmo in capo) è baciato da una Naiade. Su concessione del Comune di Parma, Civiche Collezioni d'Arte - Ufficio Patrimonio Artistico.

Crediti fotografici: Comune di Parma, Civiche Collezioni d'Arte - Ufficio Patrimonio Artistico

doppia raffigurazione della personificazione del fiume sarebbe, da un punto di vista prettamente compositivo, un vero e proprio espediente iconografico atto a guidare l'osservatore nella lettura progressiva e ordinata degli affreschi, secondo dinamiche del rapporto tra arte e spettatore indagate da John Shearman nel capitolo del suo studio sulle cupole rinascimentali. ${ }^{13}$

Nelle profondità del fiume le Naiadi hanno 'una mason che mai fu la più bella' (Inamoramento, III. 7. 8. 3), fatta di colonne di cristallo e capitelli d'oro (secondo le caratteristiche architettoniche di un

\footnotetext{
${ }^{13} \mathrm{Si}$ rimanda a John Shearman, Arte e Spettatore nel Rinascimento italiano: 'Only connect. .', trad. it. di Barbara Agosti (Milano: Sillabe, 1995), in particolare al capitolo quarto, 'Cupole', pp. 149-91, dove, non a caso, sono citate, tra le altre, opere di Correggio.
} 


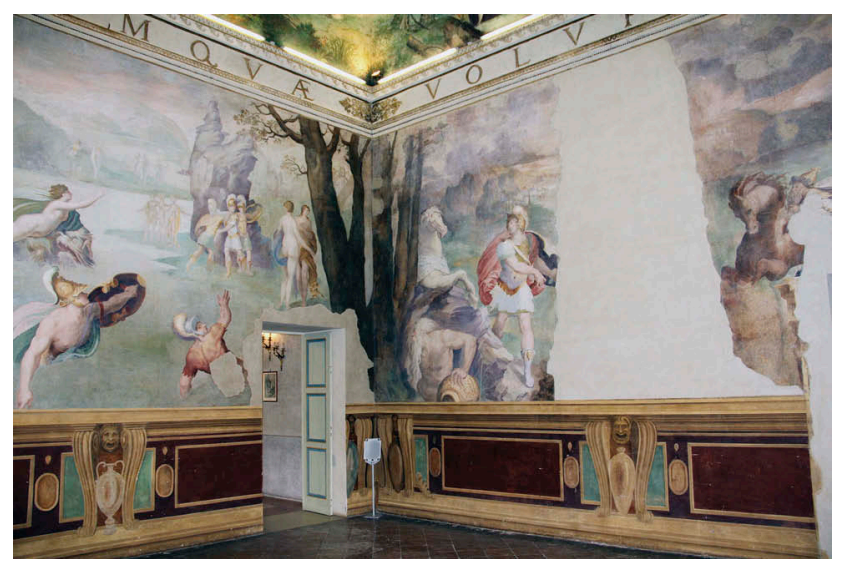

Figura 5. 'Sala del bacio', parete II: riemersione dei cavalieri dal fiume del Riso. Parete III: personificazione del fiume del Riso come foce; Orlando mentre si allontana dalla selva incantata sta per fronteggiare Gradasso; un nano sopraggiunge a cavallo. Su concessione del Comune di Parma, Civiche Collezioni d'Arte - Ufficio Patrimonio Artistico.

Crediti fotografici: Comune di Parma, Civiche Collezioni d'Arte - Ufficio Patrimonio Artistico

palazzo che però era stato già descritto in I. 8. 6 - come osservava scrupolosamente la De Vito Battaglia). ${ }^{14}$ Le ninfe acquatiche inscenano danze al suono di canti corali, baciano, baciate, 'baroni e gran milizia' (Inamoramento, III. 7. 23. 4) dalla mente e la virtù obnubilata:

A suon de trombe quivi se trescava

Zogliosa danza che di qua non si usa:

Nel contrapasso l'un l'altro basava

Né se potea tener la boca chiusa.

A cotal acto se dimenticava

Ciascun sé stesso: e io facio la scusa,

E credo che un bel baso a boca aperta

Per la dolceza ogni anima converta.

(Inamoramento, III. 7. 29)

Chi siano costoro il poeta lo ha già descritto con versi caustici, quasi misogini; cosa cerchino, è palese, pura voluttà:

Queste Naiàde nel'acqua dimorano,

Per quella solaciando come el pesce,

E per incanto gran cose lavorano,

Che ogni dissegno a lor voglia riesce;

De cavalier sovente se inamorano,

Ché star sencia om a ogni dama rincresce

(E di tal fate assai ne sono al mondo,

Ma non si vegion tutti i fiumi al fondo).

(Inamoramento, III. 7. 7)

D'altro canto, ai piaceri della vita allude la seconda iscrizione della sala, dipinta in capitali romane lungo la cornice d'imposta della volta, che interrompe il flusso continuo tra i due registri, tratta (con errore di trascrizione notato ancora una volta dalla De Vito Battaglia) da un emistichio

\footnotetext{
${ }^{14}$ De Vito Battaglia, 'Leggende cavalleresche', p. 13.
} 
virgiliano delle Bucoliche (II. 65): 'Trahit sua quemque voluptas', dall'ecloga in cui si narra di un'amicizia impossibile, come quella di Coridone e Alessi, qui citata forse esclusivamente come sentenza antiquaria. ${ }^{15}$ 'Ognuno tragga il piacere da ciò che preferisce', purché scientemente scelto, verrebbe da dire; citazione che in questo contesto però non si addice a Orlando, il cui piacere è prodotto artificialmente da un incantesimo.

Grande quadro sinottico di molteplici stanze del canto, la parte qui definita prima è l'apice della narrazione, da cui deriva il nome vulgato di 'Sala del bacio'. Capaci di grande sintesi, i due pittori, in una sola scena, condensano la climax del canto articolando la superficie da un lato con l'episodio della festa e dall'altro con quello della liberazione di Ruggero e di Orlando. Al centro della parete e sul lato destro, diafane pennellate di biacca danno corpo ai fusti di colonna di cristallo che consentono di guardare attraverso e non distorcono l'immagine, secondo una caratteristica suggerita al pittore dall'ekphrasis del palazzo dell'incantatrice Urganda nell'Amadigi di Bernardo Tasso, come ha proposto Venturelli. ${ }^{16}$ Elegantissima, a passo di danza, la pittura indugia su particolari raffinati, come ad esempio i piedi calzati di sandali all'antica che si riflettono sulla superficie specchiante dell'acqua. Si è negli abissi, quindi l'immagine è letteralmente e visivamente una mise en abîme sofisticatissima, possibile solo alla Pittura. ${ }^{17}$ Mentre Orlando è ancora intento a profondersi in baci, sulla sinistra è in atto la liberazione di Ruggero, come l'altro schiavo dei sensi, per intervento di Brandimarte edotto da Fiordelisa che gli ha rivelato, per spezzare l'incantesimo, di collocare sulla testa dei cavalieri sedotti una coroncina di erbe e fiori (Inamoramento, III. 7. 33-35; Figura 4). Dal punto di vista compositivo, all'affollamento dell'affresco sulla volta e sulla parete con il palazzo di cristallo pare facciano da contrappunto il numero ridotto di figure e la composizione più diluita delle altre tre pareti. Nella seconda parete si assiste a: esterno, superficie del fiume, tutto campo, 'rari nantes' galleggiano a pelo d'acqua non del tutto riemersi, riaffiorando 'sì come zuche' a poco a poco (Inamoramento, III. 7. 36). Tanta parete, ma poca 'historia' (Figura 5).

Come accennato in esordio, le letture iconografiche degli affreschi proposte finora si interrompono con rare eccezioni all'altezza della seconda parete, lasciando inevasa la funzione di altri soggetti che tuttora si scorgono affrescati sulle altre due (qui definite terza e quarta), purtroppo compromesse da abbondanti cadute di superficie pittorica. Assai lacunosa proprio nella parte centrale, la parete terza introduce l'immagine elegante di un cavaliere, memore del San Vitale di Parmigianino nella chiesa parmense di San Giovanni Evangelista, ${ }^{18}$ in atto di allontanarsi dalla selva incantata e dal fiume del Riso (qui ritratto come foce), nell'atto di guardare indietro, mentre sguaina il brando, inseguito da un destriero bardato, al galoppo (Figure 5, 6). Si tratta di Orlando sfidato da Gradasso per il possesso di Durindana come rivelano alcuni versi del solito canto (Inamoramento, III. 7. 42-52). In lontananza s'intravvede la cinta muraria di una città con edifici colonnati, ponti, forse una piramide con un globo al vertice (come quella in un emblema di Achille Bocchi, che anche a Venturelli pare una fonte iconografica utilizzata dai pittori) ${ }^{19}$ qui inserito in funzione meramente ornamentale. In questo punto la scena s'interrompe bruscamente. La pellicola è danneggiata da un'ampia caduta di colore, ma la parte rimanente sulla destra consente di proseguire la lettura del racconto.

\footnotetext{
${ }^{15}$ Nell'affresco si legge 'Trahetas', si veda De Vito Battaglia, 'Leggende cavalleresche', p. 14 e nota 12. 'Formosum pastor Corydon ardebat, Alexin | delicias domini nec quid speraret habebat' (ECl., II. 1-2) [Il pastore Coridone ardeva per il bell'Alessi, delizia del padrone. Ma non aveva nessuna speranza; traduzione mia]. Si fa nuovamente riferimento al verso infra, n. 22.

${ }^{16}$ Venturelli, La corte farnesiana, p. 76, dove si riportano i versi del poema al canto I. 57.

${ }^{17}$ Alla luce dei preziosismi iconografici evidenziati, sarebbe allora suggestivo rintracciare la eco della disputa sul paragone delle Arti figurative, lanciata da Benedetto Varchi a Firenze nel 1546, seguendo un percorso critico che porterebbe troppo al di là dal tema di questo saggio, della quale tuttavia i pittori non v'è dubbio siano stati a conoscenza.

${ }^{18} \mathrm{Si}$ fa riferimento all'affresco realizzato da Parmigianino nella chiesa di san Giovanni Evangelista nel 1523 ca., navata sinistra, seconda cappella, sottarco d'accesso, in situ.

${ }^{19}$ Sull'utilizzo dell'iconografia delle Simbolicae Questiones edite a Bologna nel 1555, si veda Venturelli, La corte farnesiana, pp. 164-65.
} 


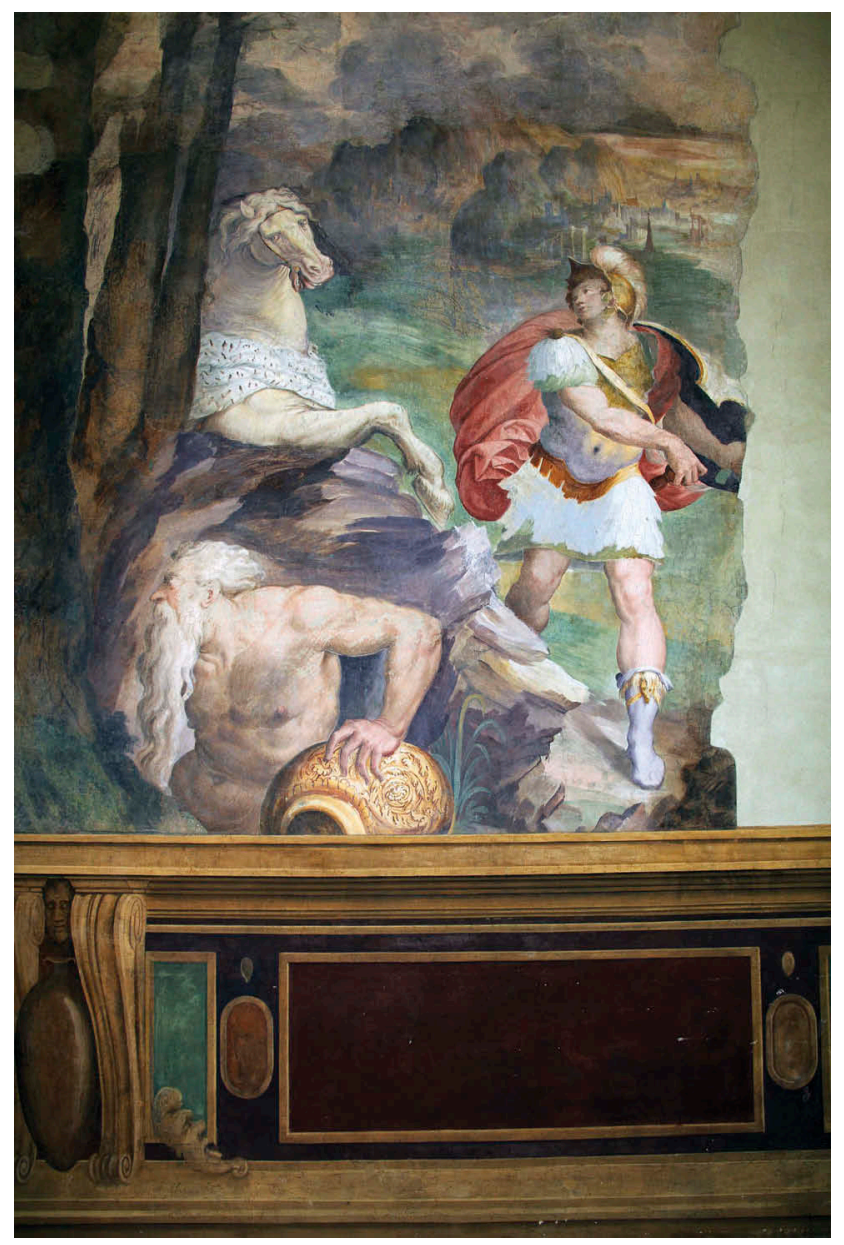

Figura 6. 'Sala del bacio', parete III (particolare): Orlando mentre si allontana dalla selva incantata sta per fronteggiare Gradasso; il fiume del Riso come foce. Su concessione del Comune di Parma, Civiche Collezioni d'Arte - Ufficio Patrimonio Artistico.

Crediti fotografici: Comune di Parma, Civiche Collezioni d'Arte - Ufficio Patrimonio Artistico

Un nano sopraggiunge a cavallo e invoca, come narra Boiardo, l'aiuto dei prodi cavalieri sfidandoli con toni risoluti sull'alto concetto dell'onore e della cavalleria (Figura 5):

'Signor, se amati la cavalaria,

Se adiffendeti el drito e la iusticia,

Fati vendetta de una fellonia

Magior del mondo e più strana nequitia!'

(Inamoramento, III. 7. 38. 1-4)

È stata rapita l'ennesima donzella, che richiede il soccorso immediato dei cavalieri, secondo un rivolo collaterale allo svolgimento del poema che si perderà nelle trame articolate degli ultimi canti. Cessato il rapporto osmotico tra testo e immagini, alla quarta e ultima parete della sala, dove campeggiano cinque personaggi non menzionati esplicitamente negli ultimi canti del poema, è demandato il compito di fornire una conclusione. Si tratta di due amanti e di due putti, distolti dai loro negozi amorosi e ludici, da una figura in volo a cavallo di un ibrido di equino e rapace, identificabile come personificazione della guerra 


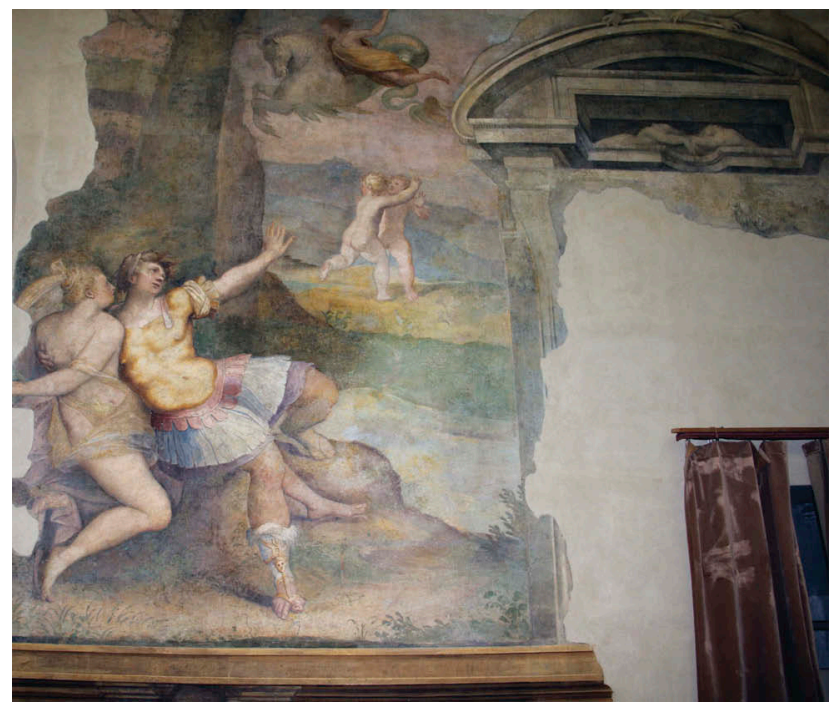

Figura 7. 'Sala del bacio', parete IV (particolare): I'irruzione della guerra distoglie dai piaceri. Su concessione del Comune di Parma, Civiche Collezioni d'Arte - Ufficio Patrimonio Artistico.

Crediti fotografici: Comune di Parma, Civiche Collezioni d'Arte - Ufficio Patrimonio Artistico

(Figura 7). Com'è noto, l'epilogo dell'Inamoramento de Orlando costituisce l'ouverture del Furioso con il roboante assedio tra sangue e bestemmie di saraceni e cristiani consumato sotto le mura di Parigi. ${ }^{20}$ La quarta parete, orientata a Est della stanza affrescata, finora trascurata, svolge in conclusione una doppia funzione affatto irrilevante. Infatti, se da un lato chiude il cerchio iconografico e narrativo fornendo un epilogo allegorico e non ecfrastico per il ciclo di affreschi; dall'altro anticipa la fonte iconografica da cui trae materia la decorazione eseguita nella sala successiva, ossia il Furioso, dei cui innumerevoli episodi è stato scelto di isolare le vicende legate ai sortilegi di Alcina, narrate nei canti quinto e sesto. ${ }^{21} \mathrm{Si}$ tratta ancora una volta di un tema legato al concetto della compromissione della virtù, qui presentato sotto il segno di un amore soggiogato, perché prodotto artificialmente per incantamento e non come conseguenza di una scelta deliberata, cui si rimedia per intervento di terzi: un tema allegorico, dunque, che allude alla necessità di non smarrire il lume della ragione cedendo alle lusinghe dei sensi e di poter fare affidamento sul nobile principio dell'amicizia, intesa ancora una volta come alleanza nelle avversità.

Primogenito di Pier Luigi e di Gerolama Orsini, consorte della figlia di Carlo V Imperatore, Margherita d'Austria, secondo duca di Castro di Parma e Piacenza, nipote del potentissimo Paolo III, il committente del palazzo Ottavio Farnese, come il fratello minore, il 'cardinal nepote' Alessandro, fu un raffinatissimo cultore delle arti, e inevitabilmente delle armi. In questo senso allora non stupisce la scelta di far raffigurare il tema dei poemi epici come celebrazione dell'alto officio della cavalleria.

Così come Alessandro, con l'aiuto di Annibal Caro, aveva costellato il palazzo pentagonale di Caprarola - dove tra il 1569 e il 1571 lo stesso Bertoja avrebbe affrescato le stanze 'della Penitenza', 'dei Giudizi' e 'dei Sogni' - di una mitopoiesi dinastica giustificata su base

\footnotetext{
${ }^{20}$ Sull'iconografia dell'assedio di Parigi in alcune edizioni illustrate del Furioso si consideri Angelo Maria Monaco, 'L'iconografia dell'assedio di Otranto e il frontespizio del Tancredi: Due episodi di fortuna iconografica delle edizioni illustrate Valgrisi e de' Franceschi del Furioso', in Le sorti di Orlando: Illustrazioni e riscritture del Furioso, a cura di Massimiliano Rossi e Daniela Caracciolo (Lucca: Pacini Fazzi, 2014), pp. 247-59.

${ }^{21}$ La 'sala di Alcina' si ritiene sia stata affrescata entro il 1563 da Girolamo Mirola, con un intervento del Bertoja nella zoccolatura decorata con putti che recano scudi di scorcio.
} 


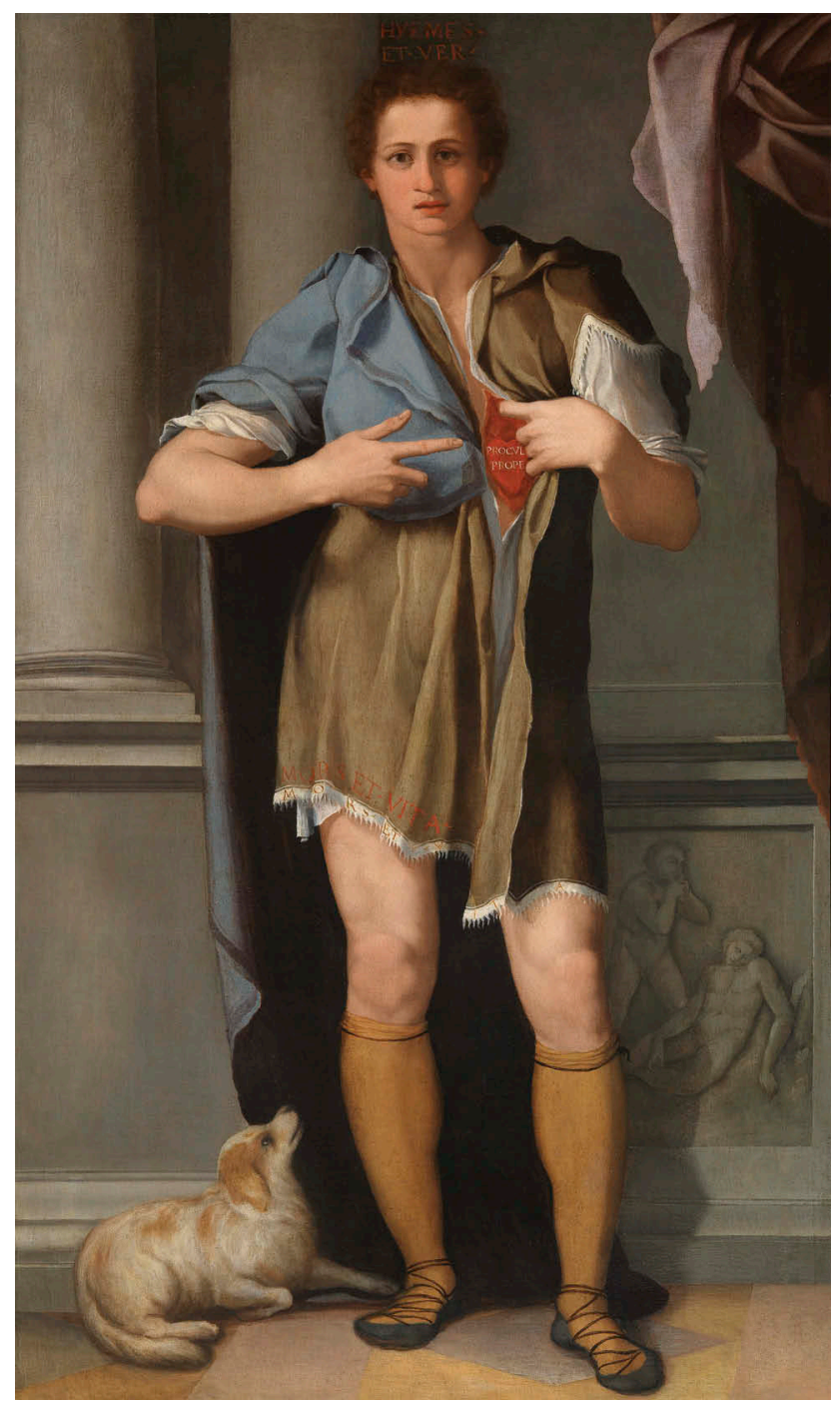

Figura 8. Mirabello Cavalori, Personificazione dell'Amicizia, collezione privata (foto dell'autore).

Crediti fotografici: Crediti fotografici dell'autore

smaccatamente ovidiana ruotante attorno e sostenuta dalla figura di Ercole, ${ }^{22}$ Ottavio, senza dubbio con il supporto di un umanista inventor del programma iconografico, tuttora al vaglio degli studi, fece ricorso a un sistema allegorico fondato su base retorica e letteraria di tipo epico. Ossia a un immaginario tanto adeguato al suo ruolo di 'uomo d'arme', cui l'aveva destinato la primogenitura, e pertanto efficace per celebrare la missione altissima della casata che si trovò a rappresentare in una congiuntura politica assai complessa, quanto di 'principe colto', raffinato esponente della 'Repubblica delle Lettere'.

In conclusione, mette conto ritornare alle premesse di questo intervento cercando di discutere la connessione, finora non affrontata, tra l'argomento del canto e il tema iconografico, ricollocando i 'baci', da cui trae il nome la stanza, in una posizione marginale rispetto al significato

\footnotetext{
${ }^{22}$ Ampia è la letteratura sul palazzo, in particolare sui componimenti ecfrastici in lode di Caprarola si veda Tommaso Casini, 'Annibal Caro a Caprarola: dal programma pittorico all'ecfrasi', in Metodo della ricerca e ricerca del metodo storia, arte, musica a confronto, a cura di Benedetto Vetere (Galatina: Congedo, 2009), pp. 203-29.
} 
complessivo veicolato dall'iconografia. Come si evince dai versi proemiali del canto settimo del libro terzo dell'Inamoramento, il tema principale di questo punto del poema è quello dell'amicizia, considerata virtù cardinale del codice etico cavalleresco cui aderiscono i protagonisti del poema. A tale virtù, fondamento della cavalleria, equiparata al diritto e alla giustizia, fa appello il senso comune dei paladini in cerca di Orlando che ha smarrito la ragione, così come il nano che invoca il loro intervento verso la fine del canto. L'Amicizia è un patto vincolante ed è un valore elogiato nel Cinquecento da letterati e pittori in una condivisa retorica ciceroniana che cela a volte sentimenti più articolati, elevati ai più alti ideali platonici, giustificati con esempi tratti dalla storia antica e dal mito:

Che giova aver de perle e de or divitia,

Aver alta possancia e grande istato

Quando si gode sol, senza amicitia?

(Inamoramento, III. 7. 2. 1-3)

Così scrive Boiardo, nella seconda stanza del canto, dopo aver dichiarato, nella prima ottava già richiamata, la natura e le peculiarità dell'Amicizia. In opposizione, nel senso di concordanza discorde, allora, risulterebbe l'epigrafe 'Trahit sua quemque voluptas', tratta dall'ecloga in cui si narra di un'amicizia impossibile, come quella di Coridone e Alessi. ${ }^{23}$ Tema assai caro ai membri della cosiddetta 'Repubblica delle Lettere', l'Amicizia è un topos rilevante dell'immaginario rinascimentale, e viene intesa sia come contratto o mercatura, sia quale Accademia ${ }^{24}$; oltre a essere rappresentata in forme iconografiche di compiutezza icastica come un 'apalesar l'un l'altro el cuore' (Inamoramento, III. 7. 1. 6). L'immagine poetica messa in versi da Boiardo, figlia di un sentire comune e di una topica codificata, non a caso diventerà vero e proprio attributo iconografico della personificazione dell'Amicizia.

In questo senso va letto, ad esempio, il dipinto di Mirabello Cavalori, in collezione privata, in cui un giovane con il petto squarciato, stante al di qua di un bassorilievo figurato in cui la critica ha riconosciuto Apollo che piange Giacinto oppure Achille e Patroloco, mostra il cuore (Figura 8). ${ }^{25}$ Così come, in conclusione, l'iconografia del medesimo concetto nell'Iconologia di Cesare Ripa, dove proprio un cuore tenuto in mano da una fanciulla 'scapigliata [...] vestita di bianco' è attributo inequivocabile di un sentimento così nobile. ${ }^{26}$

\section{Disclosure statement}

No potential conflict of interest was reported by the authors.

\footnotetext{
${ }^{23}$ Si veda supra, n. 15.

${ }^{24}$ Fumaroli, La Repubblica delle Lettere, p. 229, dove, in particolare, soffermandosi sulle affinità che tra Quattrocento e Seicento accomunano gli umanisti rendendoli membri di una medesima 'Accademia' classicamente intesa, l'autore ricorda lo stesso verso delle Bucoliche che campeggia al centro della sala del bacio: Trahit sua quemque voluptas.

${ }^{25} \mathrm{Si}$ veda Antonella Fenech Kroke e Mirabello Cavalori, 'Allegoria dell'Amicizia', in II Cinquecento a Firenze: 'Maniera moderna' e Controriforma, catalogo della mostra a cura di Carlo Falciani e Antonio Natali (Firenze, Palazzo Strozzi 21 settembre 2017-21 gennaio 2018) (Firenze: Mandragora, 2017), pp. 152-53.

${ }^{26}$ Cesare Ripa, Iconologia, a cura di Sonia Maffei, testo stabilito da Paolo Procaccioli (Torino: Einaudi, 2012), p. 31.
} 